\title{
Integration of FBG Strain Sensors in WDM Networks, Effects on Quality Factor
}

\author{
Ali Al-Lawati* and Hadj Bourdoucen \\ Department of Electrical and Computer Engineering, College of Engineering, Sultan Qaboos University, P.O. Box 33, P.C. 123, \\ Al-Khodh, Muscat, Sultanate of Oman
}

Received 12 May 2007; accepted 3 October 2007

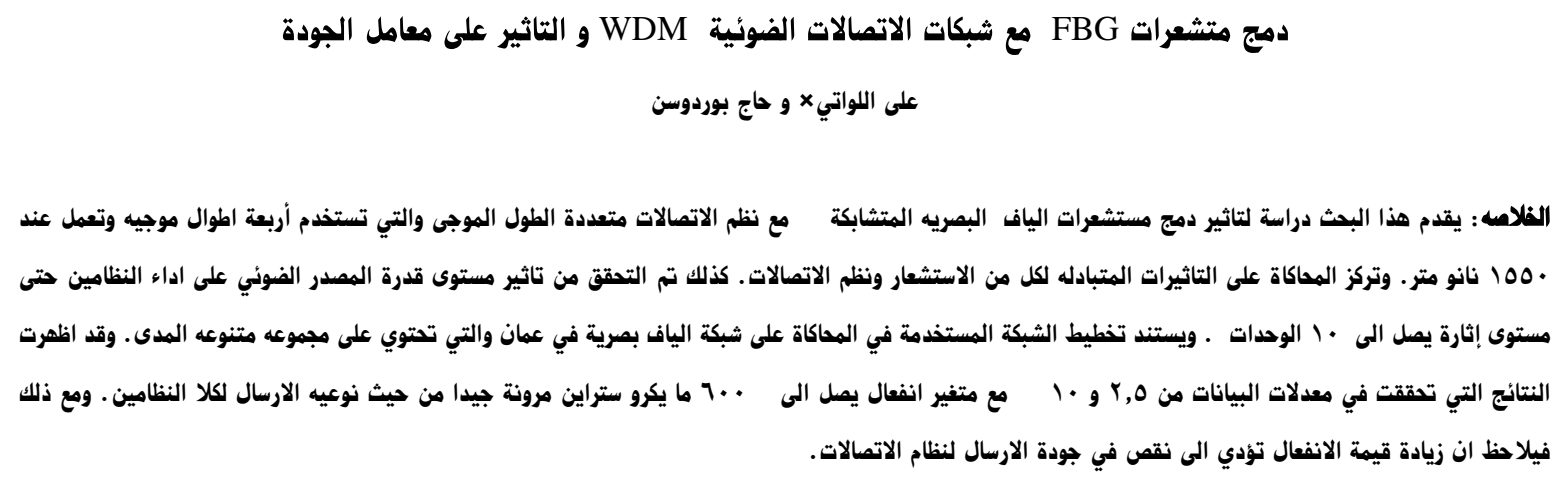

الهزرهات الهثاهية: مستشعرات الياف براج البصرية المتشابكة ، مستشعرات الالياف الضوئية، متعددة الطول الموجي ، معامل الجوده.

\begin{abstract}
A study of the effect of integrating an FBG sensor in a four wavelength WDM communications system operating at $1550 \mathrm{~nm}$ is presented. The simulations considered focus on the mutual effects of both the sensing and the communications systems. The effect of power levels of the interrogating optical source on the performance of the two systems is also investigated under excitation levels of up to $10 \mathrm{dBm}$. The network layout used in the simulations is based on an actual optical link in Oman having a variety of spans. The results obtained at data rates of 2.5 and 10 Gbps with variable strains up to $\pm 600 \mu$ s show a good tolerance in terms of quality of transmission for the two systems. However, the greater the strain values, the more noticeable are the degradations of transmission quality parameters of the communications system.
\end{abstract}

Keywords: FBG, Fiber optic sensor, WDM, Quality factor

\section{Introduction}

The attractive features of fiber optic sensors such as lack of sparking and immunity to electromagnetic fields has given it a great advantage over electronic sensors in many applications, including civil engineering, oil, gas and power generation industries (Abad, et al. 2003). In addition to its usefulness in embedded sensors applications and the inherited attractive features of optical fiber sensors, Fiber Bragg Gratings (FBGs) are driven by their performance accuracy and versatility of the utilized technology (Agarwal, 2001 and Bourdoucen and Al-Lawati, 2006).

Fiber optics communication signal carriers comprise about 95 percent of worldwide transmission capacity (Chan, et al. 1999). The introduction of the well known Wavelength Division Multiplexing (WDM) and later the Dense WDM techniques gave the fiber optic communica-

*Corresponding authors e-mail: lawati@squ.edu.om tions such a leading edge in providing astronomical bandwidth capabilities of more than 10 terabits per second on a single optical fiber. In fact, the Japanese Nippon Telegraph and Telephones Corporation has recently announced on its website reaching a data transmission rate of 14 Tbps over a $160 \mathrm{~km}$ optical fiber.

This attractive feature of WDM makes it popular for applications other than communications such as multiplexing many FBG sensors simultaneously on a single fiber. Such applications were demonstrated in numerous published articles, eg. (Abad, et al. 2003 and Fan, et al. 2004). Other multiplexing techniques to interrogate FBG sensors were also reported eg. (Gebremichael, et al. 2005 and o-eland.com/sensorproducts/sensor_strain.html).

In this paper, a study of the effect of integrating an FBG sensor was conducted in a four wavelength WDM communication system operating at $1550 \mathrm{~nm}$ region with $1 \mathrm{~nm}$ spacing. The operating wavelengths are shown in Fig. 1 below. Four wavelengths are used to study the mutual 


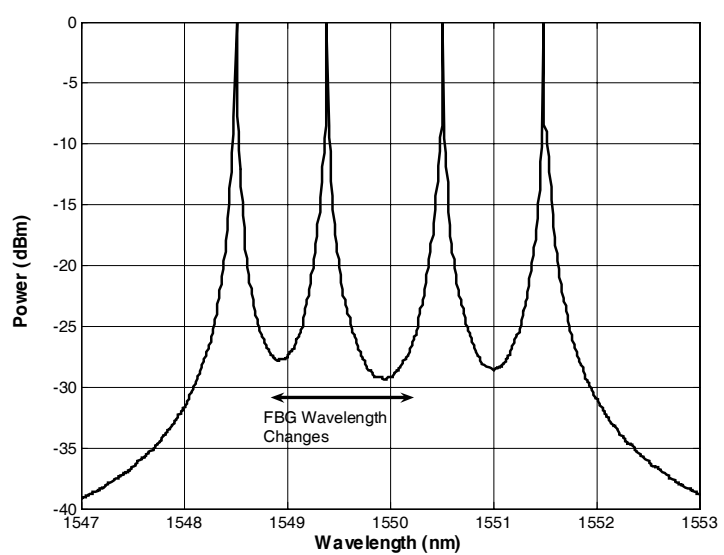

Figure 1. Spectra of the four DM sources used in simulation

effects of sensing and communications systems. These four wavelengths or channels are referred to hereafter as $\lambda_{1}, \lambda_{2}, \lambda_{3}$ and $\lambda_{4}$ corresponding to wavelength of 1548.5, $1549.5,1550.5,1551.5 \mathrm{~nm}$ respectively.

The simulations considered focus on the mutual effects on both the sensing and the communications systems. The effect of power levels of the interrogating optical source on the performance of the systems is also investigated under excitation levels of up to $10 \mathrm{dBm}$. The network layout used in the simulations is based on an actual optical link in Oman having a variety of spans operating at 2.5 and $10 \mathrm{Gbps}$. The strain sensor is located $35 \mathrm{~km}$ away from the nearest optical network access point that consists of an Optical Add and Drop Multiplexer (OADM).

\section{Theory and System Description}

The FBG sensor exhibits a shift of $\Delta \lambda_{\mathrm{g}}$ in the reflected Bragg wavelength from the central wavelength $\lambda_{\mathrm{g}}$ due to change in temperature and strain.

$\Delta \lambda_{\mathrm{g}}$ can be found using the following relation (Kang, et al. 1998):

$$
\Delta \lambda_{g}=2\left(\Lambda \frac{\partial n}{\partial l}+n \frac{\partial \Lambda}{\partial l}\right) \Delta l+2\left(\Lambda \frac{\partial n}{\partial T}+n \frac{\partial \Lambda}{\partial T}\right) \Delta T
$$

Where, $\delta l, \delta n, \delta \Delta$ and $\delta T$ are the changes in the fiber, length, index of refraction, optical grating period and temperature respectively. Assuming that the temperature effect is negligible, the Bragg wavelength shift due to a homogenous and isotropic strain on the grating can be expressed as (Lim, et al. 2001; Madhav and Asokan, 2004):

$$
\Delta \lambda g=\lambda_{g} \cdot\left(1-p_{\mathrm{e}}\right) \varepsilon
$$

Where, $p_{e}$ is the effective elastooptic coefficient of the fiber and $\varepsilon$ is the applied strain. $p_{e}$ can be found using the following equation:

$$
p_{e}=\left(n_{\text {eff }}^{2} / 2\right)\left[p_{12}-\mu\left(p_{11}+p_{12}\right)\right]
$$

Where $\mu$ is the Poisson's ratio and $p_{i j}$ are FBG fiber strain optic tensors.

The applied strain will cause a shift in the Bragg wavelength, $\lambda_{g}$ or $\lambda_{2}$ (with $99 \%$ reflectivity), towards the neighboring WDM channels $\lambda_{1}$ and $\lambda_{3}$. This in turn, will cause degradation in the communications system mainly to the immediate channels surrounding $\lambda_{2}$. Since the fiber dispersion curve is not perfectly flat and is a function of the wavelength, the effect on the neighboring channels will not be identical and therefore both surrounding channels need to be examined.

In order to study this effect, the optical network shown in Fig. 2 was used.

The sensing FBG wavelength is detected $35 \mathrm{~km}$ away from Samail city. An optical ADM is implemented to add or drop $\lambda_{2}$ to the WDM link. The interrogating signal of the FBG is sent from Muscat and the response measured at Muscat as well. The FBG sensor's dynamic range assumed in the simulations is typical and is also commercially available (Peng, et al. 2002).

Each link on the network is constructed from an SMF and a DCF fibers to compensate the chromatic dispersion at $1550 \mathrm{~nm}$. The signal attenuation in both fibers is compensated using optical amplifiers.

\section{Results and Discussion}

The system layout shown in Fig. 2, was simulated based on the well known Schrödinger. The equation was solved using the Split Step Fourier Method.

The principle of the split-step Fourier method (SSFM) technique is based on the fact that the propagation of a pulse over the full length of optical fiber is considered by dividing the total length of the fiber into small segments in such a way that changes in the envelopes of optical signals can be considered sufficiently small. Within these segments, the linear and the nonlinear operators in the Schrödinger equation can be considered to act independently of each other. Hence, the effect of propagation along the fiber segment is determined by first using nonlinear operator followed by the linear operator. In this way, the accuracy and efficiency of operator splitting techniques depend on the way discretization is done in time and spatial domains (Sinkin, et al. 2003).

As for the parameters of simulation and simplifications used, these are based on the fact that the strain is homogenous and isotopic. The factor pe in Eq. (2), which is a function of $n_{e f f}, p_{i j}$ and $\pi$, is having a numerical value of approximately 0.22 and hence this equation simplifies to $\left(1-p_{e}\right) \varepsilon=0.78 \varepsilon$. This approximation, well known in the literature was used in all simulations. The $Q$-factor is then derived from the solution of Schrodinger equation.

For a non-linear dispersive and lossy Single mode and dispersion compensated fibers used in the simulations, the evolution of the profile of a slowly varying field is obtained by solving the Schrödinger equation given by: 


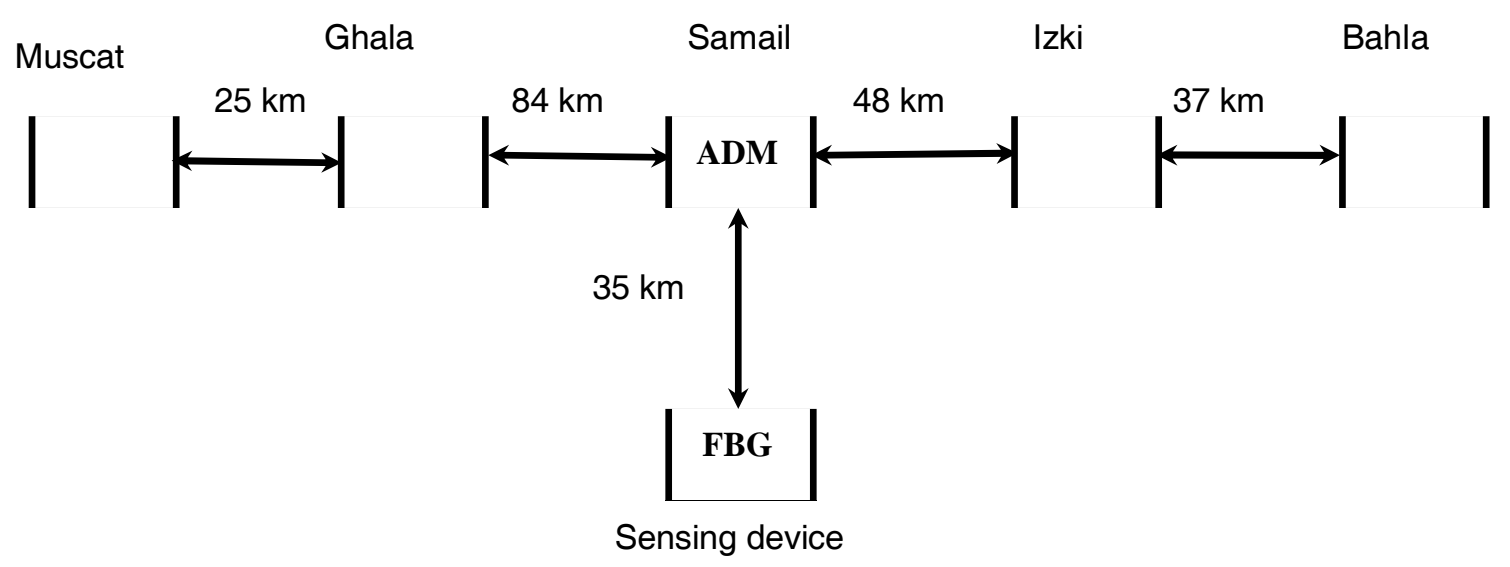

Figure 2. Physical communication system layout connecting Muscat to Bahla used in simulation to study the effect of FBG sensors on communication system

$$
\frac{\partial A}{\partial z}=-\beta_{1} \times \frac{\partial A}{\partial t}-\beta_{2} \times \frac{i}{2} \times \frac{\partial^{2} A}{\partial t^{2}}+\frac{\beta_{3}}{6} \times \frac{\partial^{3} A}{\partial t^{3}}-\frac{a}{2} A+i \gamma \times|A|^{2} \times A
$$

Where, $A$ is the amplitude of the varying electric field envelope, $\beta_{1}$ the inverse group velocity, $\beta_{2}$ and $\beta_{3}$ are the first and the second order group velocity dispersion, a is the absorption coefficient, and $\gamma$ is the nonlinear coefficient of the considered fiber. These values are taken for standard SMF and DCF fibers at a center wavelength of $1550 \mathrm{~nm}$.

Note that the $Q$ factor is approximated using mean values and standard deviations of the signal samples as indicated by relation:

$$
Q=\frac{m_{1}-m_{0}}{\sigma_{1}+\sigma_{0}}
$$

where $m_{1}, m_{0}, \sigma_{1}, \sigma_{0}$ are the mean values and standard deviations of the signal samples when a " 1 " or a " 0 " is received respectively."

In addition to these parameters, the simulations have been performed for WDM sources having constant power of $0 \mathrm{dBm}$, whereas the interrogating source used for the sensor was varied from $0 \mathrm{dBm}$ to $10 \mathrm{dBm}$. Two different bit rates were used to have a more realistic scenario transmitting at 2.5 Gbps and 10 Gbps. Figures 3 to 6 Illustrate the quality factor profiles as a function of strain for each wavelength. Figure 3, shows a flat profile of the $Q$-factor of the FBG sensor for the full range of strains considered (ie. $-600 \mu$ s to $+600 \mu s$ ) which is an indication of a good performance. This flattened behavior of the $Q$-factor seen for different power levels of the interrogating source indicates also a good tolerance to power changes. These two observations imply that integrating the sensing system for the above range of strains within a WDM communication system is viable.

However, the effects on the communications system need to be observed by examining Figs. 4, 5 and 6 corresponding to $\lambda_{1}, \lambda_{3}$ and $\lambda_{4}$ respectively.

The upper curve of Fig. 4, quasi insensitive to strain

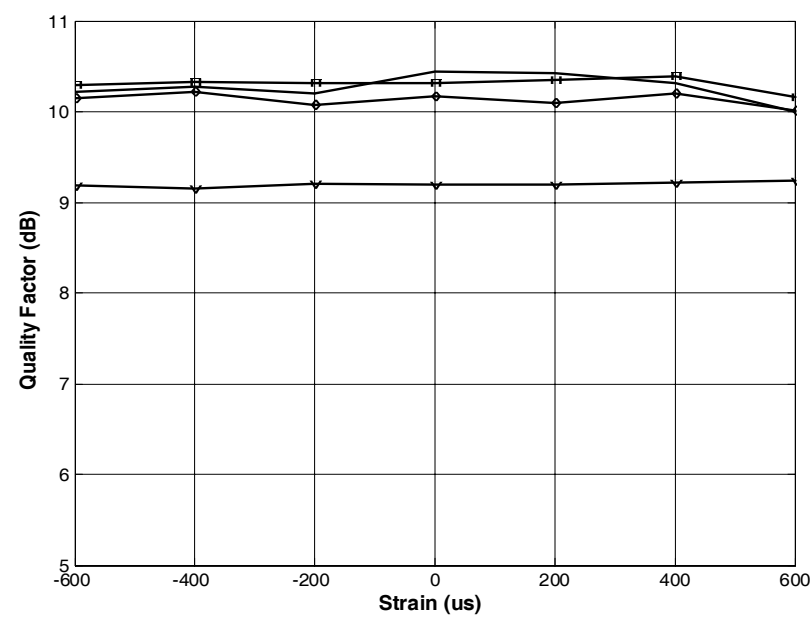

Figure 3. Quality Factor $Q$, in $d B$, for transmission performance of FBG (channel 2) operating at a data rate of $10 \mathrm{Gbps}$ for different input source power, $€: 0 \mathrm{dBm}, \mathrm{o}$ : $3 \mathrm{dBm}, \diamond: 6 \mathrm{dBm}, \nabla: 10 \mathrm{dBm}$,

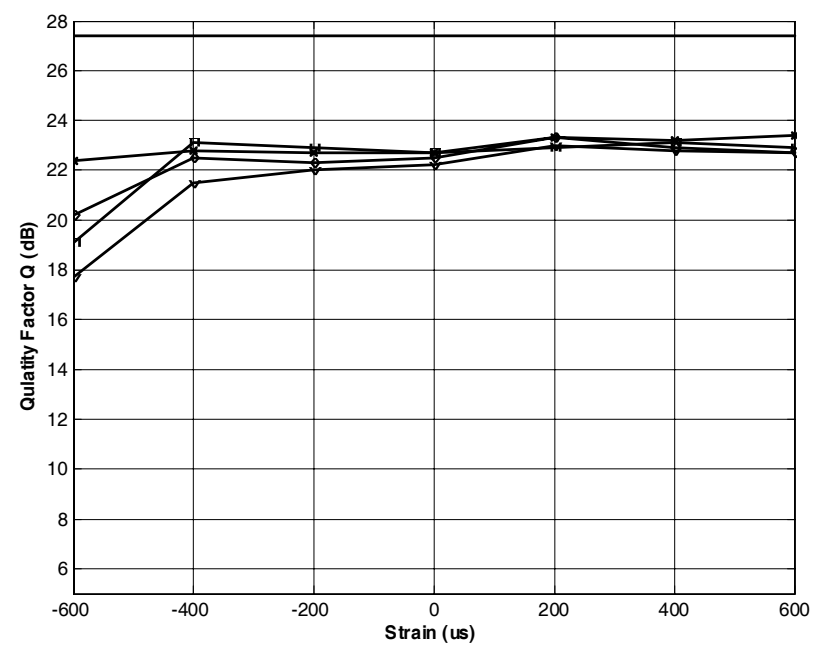

Figure 4. Quality Factor $Q$, in $d B$, for transmission performance of channel 1 (shortest wavelength on Fig. 1) operating at a data rate of 2.5 Gbps. 0: system not coupled to sensing system, €: $0 \mathrm{dBm}, \times: 3 \mathrm{dBm}, \diamond: 6$ $\mathrm{dBm}, \nabla: 10 \mathrm{dBm}$ 


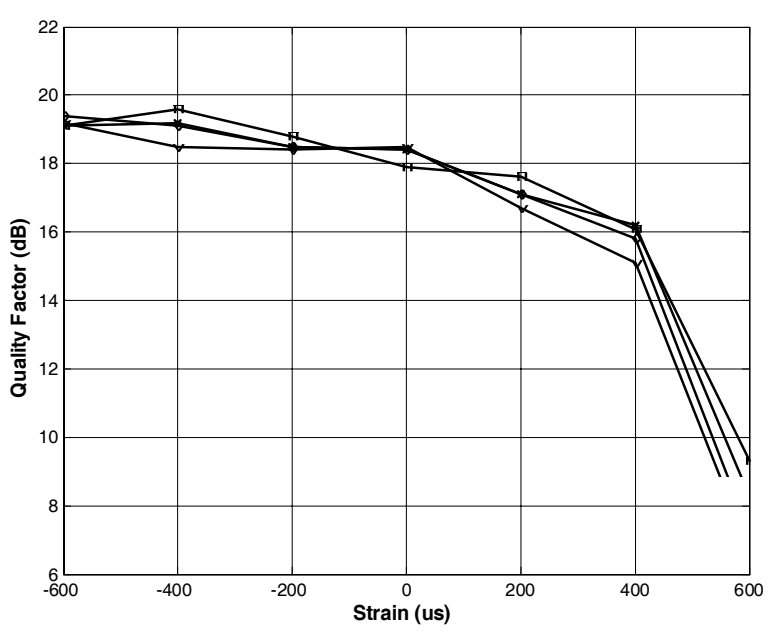

Figure 5. Quality Factor $Q$, in $d B$, for transmission performance of channel 3 operating at a data rate of 10 Gbps. 0 : system not coupled to sensing system, $€: 0 \mathrm{dBm}, x: 3$ $\mathrm{dBm}, \diamond: 6 \mathrm{dBm}, \nabla: 10 \mathrm{dBm}$

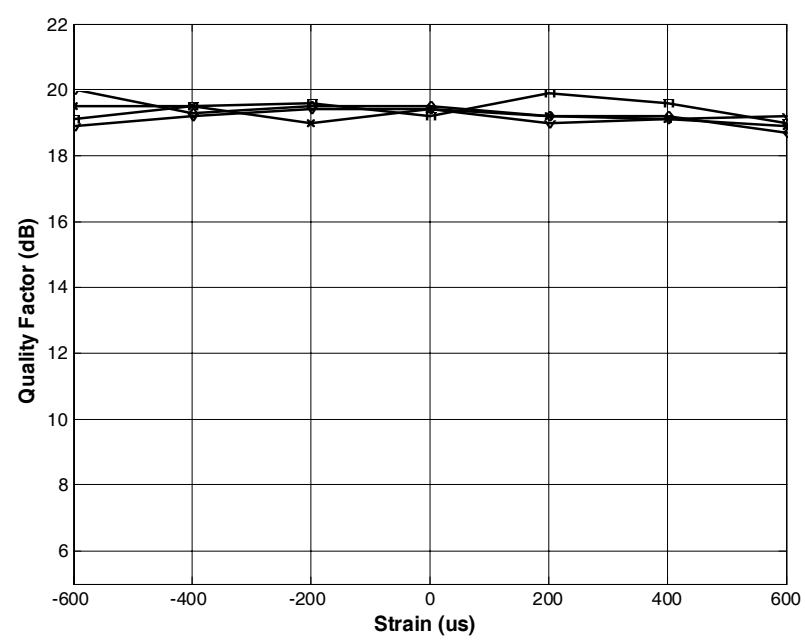

Figure 6. Quality Factor $Q$, in $d B$, for transmission performance of channel 4 operating at a data rate of 10 Gbps. 0: system not coupled to sensing system, $€: 0 \mathrm{dBm}, \times: 3$ $\mathrm{dBm}, \diamond: 6 \mathrm{dBm}, \nabla: 10 \mathrm{dBm}$,

changes, corresponds to the $Q$-factor of $\lambda_{1}$ prior to systems integration. This figure shows that integrating the sensor system causes a minor drop of the $Q$-factor. However, the overall flat response is still maintained. At strains approaching -600 $\mu$ s the curves drop slightly due to the effect of shifted $\lambda_{\mathrm{g}}$ approaching $\lambda_{1}$. The same observations are valid for Fig. 5 representing $\lambda_{3}$.

The faster drop of $Q$-factor for $\lambda_{3}$ at the neighborhood of $+600 \mu$ s is most probably due to non-uniform dispersion characteristics of the fiber and other nonlinear effects.

Figure 6 corresponds to quality of transmission of $\lambda_{4}$. As expected, due to not having $\lambda_{\mathrm{g}}$ adjacent to this wavelength, the effect on the performance of this channel is very minor. A flat response throughout the dynamic range of the sensor can be clearly observed.

Eye Diagrams for the four wavelengths at an applied strain of -200 $\mu$ s and a power of $6 \mathrm{dBm}$ for the interrogating source wavelength are shown in Fig 7. The diagrams exhibit good eye opening values for all wavelengths. The relatively wider eye opening for $\lambda_{1}$ despite having the Bragg shift towards it is mainly due to the fact that it is operating at a data rate of $2.5 \mathrm{Gbps}$, whereas the remaining three wavelengths operate at 10Gbps.
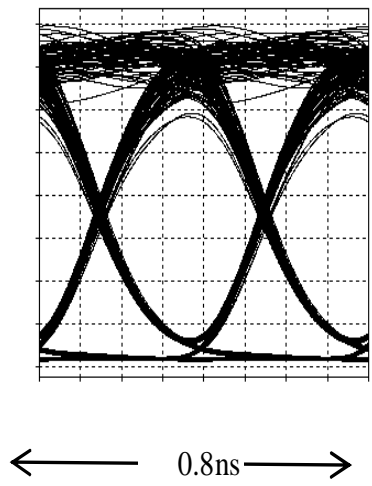

(a)

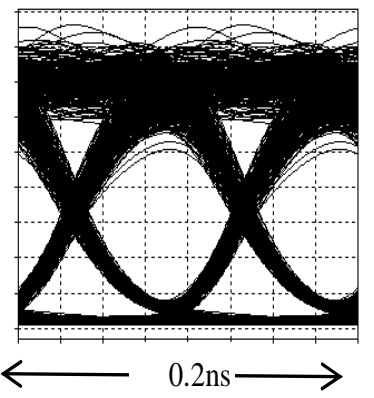

(c)

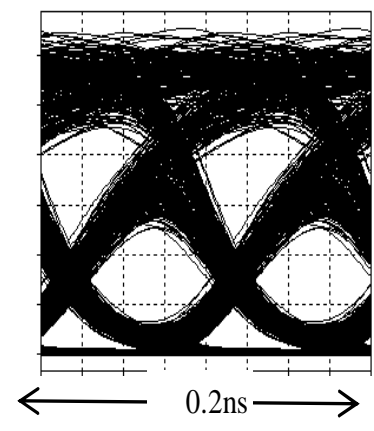

(b)

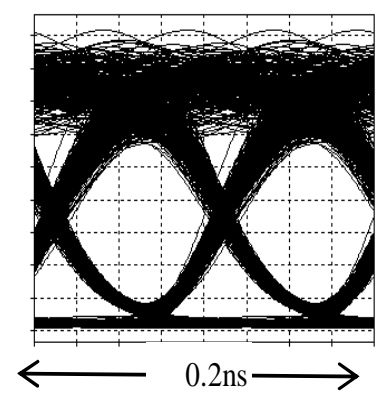

(d)
Figure 7. Eye Diagrams for the four wavelengths for an applied strain of $-200 \mu s$ and a power of $6 \mathrm{dBm}$ for the interrogating source. Eye diagrams for wavelengths 1 to 4 are respectively shown in (a through d). Note that the FBG response is shown in Fig. b) with a value of $Q$ of about $10.3 \mathrm{~dB}$

\section{Conclusions}

A study of the effect of integrating an FBG strain sensor in a four wavelength WDM communication system operating at $1550 \mathrm{~nm}$ region is presented. The simulations considered focused on mutual effects of both systems. The effect of power levels of the interrogating optical source on the quality factor of the two systems has also been investigated considering a network layout in Oman having variety of spans.

The simulations performed on the systems at data rates of 10 Gbps and 2.5 Gbps for strains up to $\pm 600 \mu$ s, exhibit a good tolerance for the integration operation. The obtained quality factors and eye diagrams for all wavelengths used and at different interrogating sensor source values, suggest that the integration has a minor effect on 
both systems. This suggests the possibility of utilizing existing communications systems for FBG sensing applications.

\section{References}

Abad, S., Araújo F. M., Ferreira, L. A., Santos, J. L. and López-Amo, M., 2003, "Interrogation of Wavelength Multiplexed Fiber Bragg Gratings Using Spectral Filtering and Amplitude-to-Phase Optical Conversion", J. Lightwave Technol., Vol. 21(1), pp 127-131.

Agrawal, G., 2001, "Applications of Non Linear Fiber Optics", Academic Press.

Bourdoucen, H. and Al Lawati, A., 2006, "Effect of Dispersion Compensation on FBG Sensing Systems Performance", J. of Instruments and Experimental Techniques, (Springer), Vol. 49(3), pp. 403-407.

Chan, P., Gong, W.J.M. and Demokan, M., 1999, "Multiplexing of Fiber Bragg Grating Sensors Using an FMCW Technique", IEEE Photonics Technology Letters, Vol. 11(11), pp. 1470-1472.

Fan, Y. and Kahrizi, M., 2004, "Applicability of an Optimized Fiber Optic Smart Structure", Proceedings of the International Conference on MEMS, NANOs and Smart Structures (ICMENS'04), pp. 386-392.

Gebremichael, Y. M., Li W., Meggitt, B. T., Boyle, W. J. O., Grattan, K. T. V., McKinley, B., Boswell, L. F., Aarnes, K. A., Aasen, S. E., Tynes, B., Fonjallaz, Y. and Triantafillou, T., 2005, "A Field Deployable,
Multiplexed Bragg Gratings Sensor System Used in an Extensive Highway Bridge Monitoring Tests", IEEE Sensors Journal, Vol. 5(3), pp. 510-519.

Kang, S., Kim, S., Lee, S., Kwon, S., Choi, S. and Lee, B., 1998, "Temperature-Independent Strain Sensor System Using a Tilted Fiber Bragg Grating Demodulator ", IEEE Photonics Technology Letters, Vol. 10(10), p. 146.

Lim, J., Yang, Q., Jones, B. and Jackson, P., 2001, "Strain and Temperature Sensors using Multimode Optical Fiber Bragg Gratings and Correlation Signal Processing", Technology Conference, Budapest, Hungary, pp. 1463-1466.

Madhav, K. and Asokan, S., 2004, "Spectrum Estimation by Wavelength Shift Time-Stamping in a Fiber Bragg Grating Sensor", IEEE Photonics Technology Letters, Vol. 16(5), p. 1355.

Peng, P., Tseng, H. and Chi, S., 2002, "Self-Healing Fibre Grating Sensor System using Tunable Multiport Fibre Laser Scheme for Intensity and Wavelength Division Multiplexing", ELECTRONICS LETERS, Vol. 38(24), pp. 1510-1512.

Sinkin, V., Holzlohner, R., Zweck, J. and Menyuk, C.R., 2003, "Optimization of the Split-Step Fourier Method in Modeling Optical-Fiber Communication Systems," J. Lightwave Technol., Vol. 21, pp. 61-68.

Warf, B., 2006, "The Professional Geographer", Vol. 58(1), pp. 1-11.

www.o-eland.com/SensorProducts/sensor_strain.html. 